\title{
Paclitaxel and spirulina co-loaded polymeric nanoparticles: in-vitro and in-vivo anticancer study
}

\author{
Yamuna Ravikumar, Dorcas Pinky Catherine, Keerthi G. S Nair, \\ Akshya Sekar, Ramaiyan Velmurugan ${ }^{\circledR *}$ \\ School of Pharmaceutical Sciences, Vels Institute of Science \\ Technology and Advanced Studies, Chennai, India.
}

\begin{abstract}
Paclitaxel spirulina nanoparticles were said to have promising anticancer activity against gastric cancer. Nanoparticles of paclitaxel-spirulina were prepared for treating gastric cancer using precipitation technique. The synergistic anticancer efficiency againstMKN45 cells retains when the paclitaxel and spirulina were encapsulated into nanoparticles. To increase the site specific delivery, intra-tumoral administration was carried in the in vivo evaluation. There was an increase in overall survival in an MKN45-transplanted mice model and notable improvement in anti-tumour efficacy when paclitaxelspirulina nanoparticles were delivered through intra-tumoral administration. The further investigation of overall anticancer mechanism of these nanoparticles is made as a major part in this research. Hence, the conjecture of this research is that, the paclitaxel-spirulina encapsulated nanoparticles could be an effective chemotherapeutic formulation for gastric cancer.
\end{abstract}

Keywords: Paclitaxel. Spirulina. Nanoparticle. In-vitro. In-vivo. Gastric cancer.

\section{INTRODUCTION}

Worldwide gastric cancers are in the fourth leading cause of cancer related deaths. The most frequent and life threatening form of the metastasis and recurrence in patients with gastric cancer is the peritoneal dissemination (Jemal et al., 2011, Isobe et al., 2011). The systemic chemotherapy regimens which are currently active for gastric cancer are not up to the mark. The researches in the Drug delivery system is mainly aiming to the increase in the penetration of the drugs in to the tumours and prolonging the drug retention in the peritoneal cavity (Yamada et al., 2008, Bajaj, Yeo, 2010).

Paclitaxel is one among the most commonly used anticancer agents which has manifested various activities against many number of tumours. The

*Correspondence: R. Velmurugan. School of Pharmaceutical Sciences. Vels Institute of Science. Technology and Advanced Studies. Chennai -600117. Cellular:+919500442754. Email: ramaiyan.dr@gmail.com cytotoxic effect of paclitaxel is achieved by G2-M cell arrest, through depolymerisation of microtubule that are accelerated from the tubulin (Diaz, Andreu, 1993). As the antitumour effect of paclitaxel is being accompanied with severe side effects due to the special solvents (Cremophor $\mathrm{EL}^{\circledR}$ ) (Dorr, 1994; Bissery et al., 1995) and the other nonspecific effects, there arose a need of specific new strategies to increase the anticancer efficacy of paclitaxel.

Spirulina platensis, a blue-green alga that consisting of hypocholestrolemic property is used as a dietary supplement. It is also a potent antioxidant and antiproliferative agent as it is a rich source of tetrapyrollic compounds that are similar to the bilirubin molecule.

Though the systemic administration failed to meet the desired concentration in tumour,there was an increase in the antitumour effect in comparison with the reduced side effects (Li et al., 2008; Maeda, 2001). The effective way to eradicate tumour is the local-regional chemotherapy.In comparison with the free drugs, the drug loaded nanoparticles are analysed by the controlled 
release of the drug,that can overcome the decrease in the drug retention in the tumour and that delivers a result of increasing anticancer effect when combined with the intra-tumoral administration.The work has been carried out in the cisplatin-loaded nanoparticles which manifested the increased antitumour effect against liver cells through intra-tumoural delivery (Idani, 2000). As there is a correlation in the effect of paclitaxel with the antioxidant capacity,there is a synergestic antitumour effect when paclitaxel and spirulina are co-deliveried. Based on the above analysis, it is manifested that, the co-administrtion of encapsulated paclitaxel-spirulina cause increased intracellular reactive oxygen species induction,by inhibiting the reactive oxygen speciesdependent Akt-pathway and activating the apoptotic pathway which increases the cytotoxic effect of paclitaxel (Li et al., 2012). This study aimed for the invitro and in vivo efficacy of the encapsulated paclitaxel-spirulilna nanoparticle site designed for gastric cancer.

\section{MATERIAL AND METHODS}

\section{Material}

Thecellswererefinedin 1640mediumwith $10 \%$ foetal bovine serum and $100 \mathrm{U} / \mathrm{mL}$ penicillin-streptomycin at $37^{\circ} \mathrm{C}$ out of a water soaked air with $5 \%$ carbon dioxide. Spirulina, paclitaxel, 3-(4,5-dimenthylthiazol-2-yl)- 2, 5-diphenyltetrazolium bromide (MTT) were purchased from Sigma-Aldrich Corporation. Gastric tumor cell line MKN45 was acquired from the Amala cancer research centre, Kerala, India. The various chemicals made used were of analytical grade.

\section{Formulation of nanoparticles}

Paclitaxel and spirulina co-stacked nanoparticles were set up by a nanoprecipitation technique. Briefly, various methoxy PEG-PCL inhibits copolymers and spirulina and paclitaxel were deliquesced in an aliquot of acetone. At room temperature the gathered natural arrangement was included drop by drop into ten different volumes of distilled water and delicately blended. For the total expulsion of acetone dialysis layer spectra POR2, (Spectrum Lab, Houston, TX) (sub-atomic weight cut off $12 \mathrm{kDa}$ ) was utilized and the arrangement was dialysed. Keeping in mind the end goal to dispose of non-retained medications and copolymer totals, the came about pale blue dissolvable was purged through a $0.22-\mu \mathrm{M}$ measured film channel (GVWP04700; Millipore, Billerica, MA). Medication free nano particles were framed in a same way by discarding the medications. For included application, void nanoparticles and arrangements of medication stacked nanoparticles were lyophilized. 1:5 is the sustaining proportion of nanoparticles with Pluronic ${ }^{\circledR}$ F68 (Sigma Aldrich, India) as the cryoprotectant.

\section{Characterization of nanoparticles}

\section{Particle size and Zeta potential}

Using photon correlation spectroscopy, the average diameter and distributed size were measured afore lyophilisation (dynamic light scattering) (BI-9000AT; Brookhaven Instruments Corporation, Holtsville, NY). Using laser Doppler anemometry, zeta potential was measured (Zeta Plus; Brookhaven Instruments).

\section{Scanning Electron Microscopy (SEM)}

Surface morphological evaluation of paclitaxel and spirulina Co-Loaded Polymeric Nanoparticles was carried out by scanning electron microscope (EVO, ZEISS Germany) for which lyophilized powder samples were spread on the carbon adhesive sample holder and coated with gold, used for scanning electron microscope.

\section{Drug Loading capacity and Entrapment Efficiency}

A LC-10 AD HPLC system (Shimadzu Corporation, Tokyo, Japan) fixed with an Agilent $\mathrm{C}-18,5 \mu \mathrm{M}, 200 \mathrm{~mm} \times 4.6 \mathrm{~mm}$ reversed-phase highperformance liquid chromatography analytical column (Agilent Technologies, Santa Clara, CA) and with a Shimadzu ultraviolet detector to examine the paclitaxel fixation. Acetonitrile/twofold distilled water (58/42 volume/volume) which have a flow rate of $1.0 \mathrm{~mL} /$ minute and decided wavelength of $228 \mathrm{~nm}$ is considered as the mobile phase of identifying paclitaxel. In view of the peak region at 7.3 minutes of retention time, the concentration of paclitaxel was controlled by a calibration curve as reference.

HPLC was performed utilizing a reversed phase Discovery BIO Widepore C5 (Supelco, Sigma Aldrich) column $(250 \times 4.6 \mathrm{~mm}$ i.d.) stuffed with $5 \mu \mathrm{m}$ permeable silica particles (300 angstrom pore breadth). This column was worked at a flow rate of $1 \mathrm{~mL} \mathrm{~min}^{-1}$ for 
ideal detachment effectiveness. All the solutions were separated through $0.5 \mu \mathrm{m}$ film filter and degassed by rising with helium before utilize. Enhancement of chromatographic partitions was performed utilizing an Alliance framework (Waters) with 2695 detachment module with auto-sampler comprising of a Waters 2998 Photo Diode-Array locator (PDA) and Waters 2475 Multi $\lambda$ fluorescence identifier. Excitation and discharge wavelength for fluorescence indicator was set at 580 and $640 \mathrm{~nm}$. The Discovery BIO Widepore C5 segment was pre-equilibrated with $20 \%(\mathrm{v} / \mathrm{v})$ fluid acetonitrile (ACN) arrangement containing $0.1 \% \quad(\mathrm{v} / \mathrm{v})$ trifluoroacetic corrosive (TFA). Twenty $\mu \mathrm{l}$ test $\left(200 \mu \mathrm{g} \mathrm{mL} \mathrm{mL}^{-1}\right)$ was infused and elution was performed utilizing a straight angle from 20 to $100 \%(\mathrm{v} / \mathrm{v}$ ) fluid $\mathrm{ACN}$ (containing $0.1 \%$ TFA) in $45 \mathrm{~min}$. Both PDA and fluorescence identifier were associated in arrangement for the identification of biliprotein subunits which uncovered two noteworthy crests at 25.612 and $27.024 \mathrm{~min}$.

The consecutive equations were used to determine DL and EE:

DL $(\%)=$ Drug's weight in nanoparticles/ weight of the nanoparticles $\times 100$

$\mathrm{EE}(\%)=$ Drug's weight in nanoparticles /weight of the feeding drugs $\times 100$

\section{In vitro cytotoxicity studies}

MTT reduction assay was utilized to ponder the in vitro drug prompted cytotoxic impacts. Density of 10,000 MKN45 cells for every well were seeded and a series of doses of free paclitaxel alone and paclitaxel \& spirulina nanoparticles joined with dimethyl sulfoxide were administered quickly at $37{ }^{\circ} \mathrm{C}$ as a negative control. After treatment, include $0.1 \%$ of MTT in each well and it is brooded for 4 hours at $37^{\circ} \mathrm{C}$.After reoving the medium,Dimethyl sulfoxide $(200 \mu \mathrm{L})$ was added to solubilize the MTT-formazan item. By utilizing the multi well spectrophotometer, absorbance at $570 \mathrm{~nm}$ was estimated. The level of the untreated controls was computed as development restraint.

\section{Evaluation of Anti-tumor effect and Survival analysis}

Mice of Male Wistar albino strains og 6-8 weeks that are 18-22 $\mathrm{g}$ weight were purchased from the animal house of Tamilnadu Veterinary and Animal Sciences University, Chennai, India and are housed in the pathogen-free facility for in vivo experiments. The local ethics committee controls and approves all the animal procedures that are to be carried out based on the guidelines. The animal house at $22{ }^{\circ} \mathrm{C}$ with a relative humidity of $40 \%-60 \%$ and a 12 -hour light $/ 12$ hour dark cycle is suitable for rising of mice. The invitro culture of MKN45 cells were gathered and it is balanced in $0.9 \%$ saline. At the mammary fat pad near the left armpit, subcutaneous administration of $1 \times 106$ live MKN45 were injected in the each mouse. The xenografts were noticeable 7 days after infusion of the cells.

Tumour Volume $(\mathrm{V})=1 / 2\left(\right.$ length $\times$ width $\left.^{2}\right)$

Day 11:57 mice with privately segregated tumors (breadth of 5.5-6.5) were selected, which are then evaluated for tumor inhibition by dividing them into three random groups. $0.4 \mathrm{~mL}$ of saline solutions containing paclitaxel alone and paclitaxel \& spirulina nanoparticle, having an equivalent paclitaxel doses were injected via tail vein of mice.

Day 13: 75 mice with privately segregated tumors (breadth of 6.5-7.4 $\mathrm{mm}$ ) and divided into three groups and the treatment was carried similarly.

Day 75: The survival rate of the mouse was recorded daily and censored.

\section{Hematoxylin and eosin staining of tumor slices}

The tumor cuts were put in $4 \%$ dried out paraformaldehyde that is sectioned $(4 \mu \mathrm{m}$ in thickness and recolored utilizing hematoxylin and eosin (SigmaAldrich Inc). Finally, the cuts were analyzed by optical microscopy.

\section{Statistical analysis}

The statistical data were represented as mean \pm standard deviation and SPSS version 16.0 were used to calculate. One-way analysis of variance was used to test the different groups in comparison of data. Survival plots were created using Kaplan-Meier and the longrank test was useful in comparing the survival curves. $\mathrm{P}<0.05$ was considered to be statistically significant. 


\section{RESULTS}

\section{Particle size, Polydispersity index and Zeta potential measurement}

The mean particle size of the nanoparticle was 198 $\mathrm{nm}$ with a polydispersity index of $0.194 \pm 0.025$. The Zeta potential of the nanoparticle was observed to be -
$25 \mathrm{mV}$, and it is adequately high to form stable colloidal nanosuspension. The image of particle size and zeta potential were represented in figure 1. It was detected that maximal DL and EE, $98.4 \%$ and $15.3 \%$ respectively were attained with optimized assay conditions of polymer concentration, surfactant concentration and stirring time.
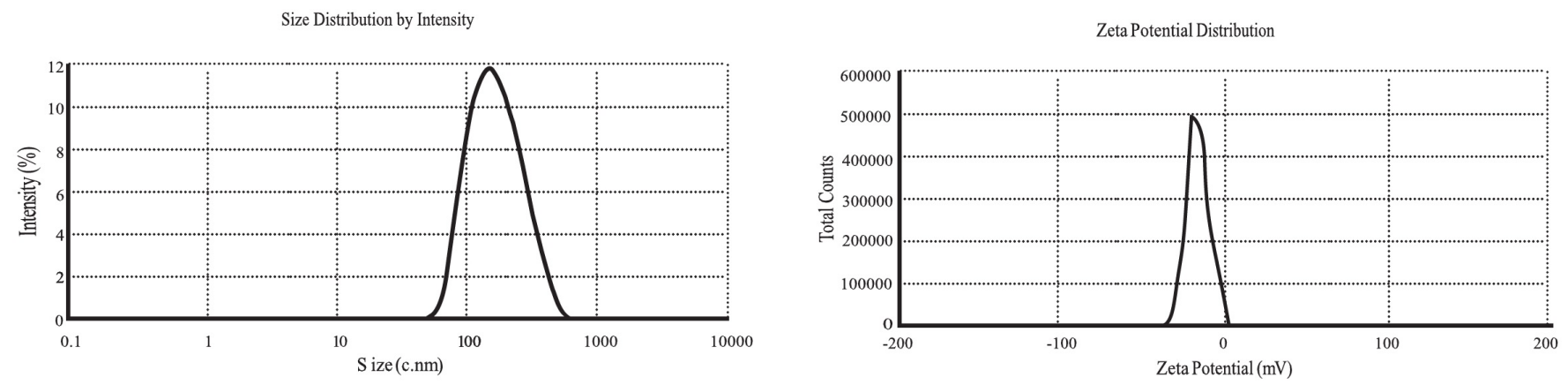

FIGURE 1 - Particle size distribution and Zeta potential measurement of paclitaxel and spirulina nanoparticles measured by DLS. The diameter of $70 \%$ nanoparticles ranged from 80 to $620 \mathrm{~nm}$. The average diameter was $198 \pm 11.2 \mathrm{~nm}$. The zeta potential recorded was $-25 \mathrm{mV}$. (Scale bar $100 \mathrm{~nm}$ ).

\section{SEM Analysis}

SEM image (figure 2) shows that the optimized formulation is nearly spherical in shape. SEM images would give a better understanding of the real geometric size of the particles and the correlation between process variables and particle size would be seen on a qualitative basis as well.

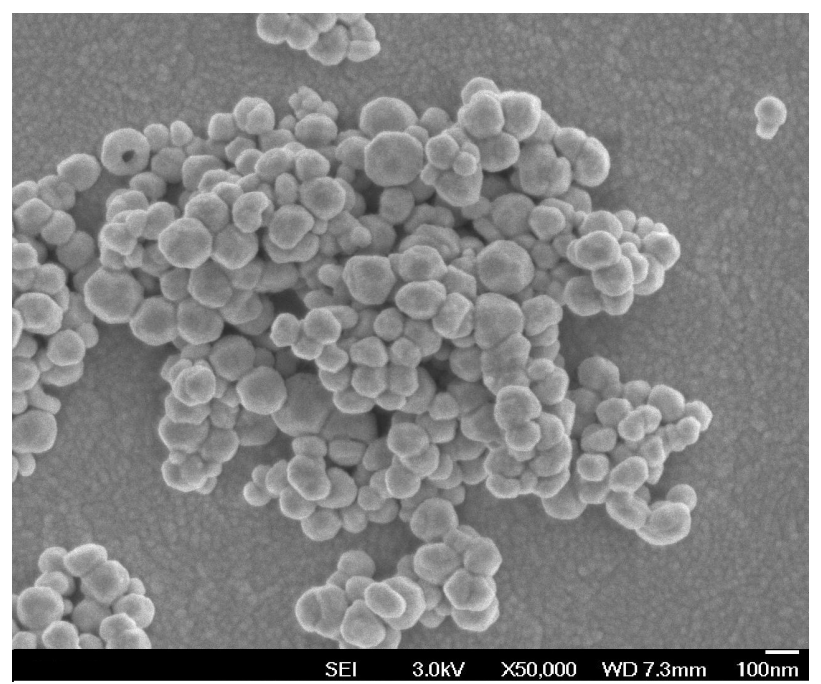

FIGURE 2 - Morphology of paclitaxel and spirulina, shown by SEM imaging. Most paclitaxel and spirulina nanoparticles are circular or elliptical. 


\section{Anti-cancer efficacy of paclitaxel \& spirulina nanoparticles against $\mathrm{H} 22$ cells}

With restraint rate, $15 \%$ at an expanded convergence of $800 \mu \mathrm{g} / \mathrm{mL}$, clear nanoparticles were shown to be about nontoxic to MKN45 cells, in the in vitro cytotoxicity test. A nontoxic measurement of $2 \mu \mathrm{M}$ is considered as a settled dosage for spirulina. Even at the equivalent doses of paclitaxel, the nanoparticles were considered to be more cytotoxic than the free paclitaxel.

The paclitaxel in cells receiving the paclitaxel and spirulina nanoparticles, the half maximal inhibitory concentration value was found as $11.07 \pm 0.97 \mathrm{nM}$ and the cells receiving free paclitaxel was found as $18.50 \pm$ $1.35 \mathrm{nM}$. Here the Nanoparticles was significantly lower when compared to the pure drug.

\section{In vivo tumor inhibition study}

On day 12 , the sizes and weights of the mouse were analysed to evaluate the tumor inhibition rate (TIR) and then they were sacrificed. On comparing the antitumour effect of the free paclitaxel and the paclitaxel-spirulina nanoparticles, the nanoparticles were proven to be superior than the paclitaxel alone $(\mathrm{P}<0.05)$.The TIR values were calculated based on size and weight. The size based values for paclitaxel and the nanoparticles are $39.8 \%$ and $\& 3.2 \%$, respectively and the weight based values are $22.2 \%$ and $62.8 \%$ (Figure 3 ). The volume and net weight changes of MKN45 xenograft tumors on day 12 after initial treatment is given in table I. In comparison, paclitaxel-spirulina nanoparticles were considered to have the superior antitumor effect than the paclitaxel.

TABLE I - MKN45 xenograft tumors on day 12 after initial treatment (Volume \& Net weight)

\begin{tabular}{|c|c|c|c|c|c|c|c|c|}
\hline \multirow{2}{*}{ Antibiotic } & \multirow{2}{*}{$\begin{array}{c}\text { ATC } \\
\text { Classification }\end{array}$} & \multicolumn{7}{|c|}{ DDD/100 bed-days } \\
\hline & & 2012 & 2013 & 2014 & 2015 & 2016 & 2017 & Total \\
\hline Ampicillin & J01CA01 & 9.300 & 13.355 & 6.746 & 5.727 & 0.024 & 5.442 & 40.594 \\
\hline Amikacin & J01GB06 & 5.449 & 10.880 & 9.579 & 9.943 & 23.48 & 27.441 & 86.772 \\
\hline Piperacillin-Tazobactam & J01CR05 & 3.326 & 8.843 & 7.387 & 2.596 & 4.619 & 3.541 & 30.312 \\
\hline Cefoperazone & J01MA02 & 0 & 0 & 1.131 & 5.052 & 5.454 & 0.180 & 11.817 \\
\hline Cefuroxime & J01DC02 & 1.286 & 0.998 & 1.988 & 2.635 & 0.424 & 0.697 & 8.028 \\
\hline $\begin{array}{l}\text { Cefoperazone - } \\
\text { Sulbactam }\end{array}$ & J01DD62 & 0.017 & 0 & 6.386 & 0.531 & 1.082 & 0 & 8.016 \\
\hline $\begin{array}{l}\text { Amoxicillin - } \\
\text { Clavulanic acid }\end{array}$ & J01CR02 & 0.140 & 0.180 & 0.5 & 0.014 & 0.714 & 0.107 & 1.655 \\
\hline Cefotaxime & J01DD01 & 2.133 & 4.229 & 0.094 & 1.219 & 0.171 & 0.421 & 8.267 \\
\hline
\end{tabular}


TABLE I - MKN45 xenograft tumors on day 12 after initial treatment (Volume \& Net weight)

\begin{tabular}{|c|c|c|c|c|c|c|c|c|}
\hline \multirow{2}{*}{ Antibiotic } & \multirow{2}{*}{$\begin{array}{c}\text { ATC } \\
\text { Classification }\end{array}$} & \multicolumn{7}{|c|}{ DDD/100 bed-days } \\
\hline & & 2012 & 2013 & 2014 & 2015 & 2016 & 2017 & Total \\
\hline $\begin{array}{l}\text { Trimethoprim- } \\
\text { Sulfamethoxazole }\end{array}$ & J01EE01 & 0 & 11.590 & 3.896 & 0 & 0.195 & 0.958 & 16.639 \\
\hline Vancomycin & J01XA01 & 2.0066 & 13.379 & 9.280 & 11.779 & 9.786 & 6.119 & 52.3496 \\
\hline Ciprofloxacin & J01MA02 & 6.174 & 10.430 & 10.806 & 4.082 & 5.240 & 0.131 & 36.863 \\
\hline Erythromycin & J01FA01 & 0 & 1.993 & 2.495 & 0.143 & 0.419 & 0.618 & 5.668 \\
\hline Metronidazole & J01XD01 & 0.326 & 1.029 & 0.324 & 0.929 & 0.290 & 0 & 2.898 \\
\hline
\end{tabular}

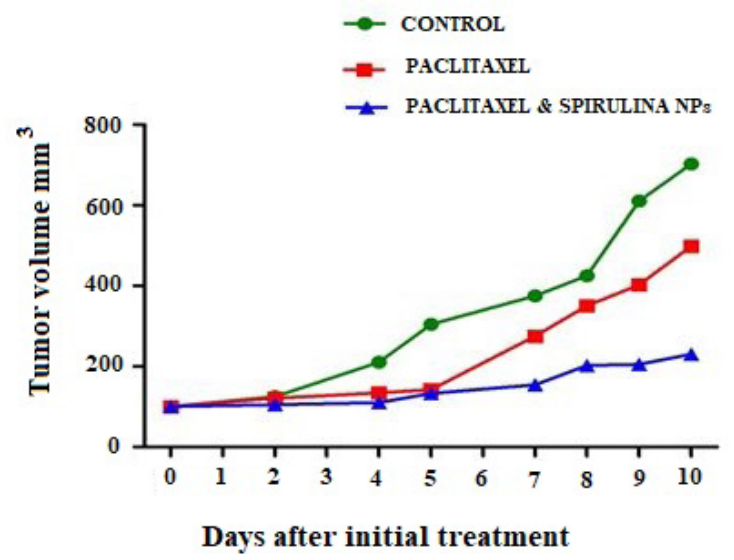

FIGURE 3 - Xenograft tumors volume change with respect to function of time. Relative tumor volume versus time is displayed.

\section{Survival analysis}

For the survival analysis, all the 75 mice were divided into three groups randomly. For every 2 days, subcutaneous tumors were measured. The survival analysis were censored on the day 75 , where all the $\mathrm{m}$ ice in the control and paclitaxel have died but three were alive in the nanoparticle group. The KaplanMeier survival curve is shown in figure 4 . There is a significant difference in the survival time between the nanoparticles and the other group and the same is presented in table II. Hence, it is known that there is an effective antitumor action in the paclitaxel-spirulina nanoparticles. 
There was a delayed growth of cancer xenocrafts in mice after the administration of paclitaxel. After treatment with the paclitaxel and spirulina nanoparticles and the equal measurements of paclitaxel of $20 \mathrm{mg} / \mathrm{kg}$, enthusiastically, a portion of the mice experienced about entire lament of the xenograft, which infers that the composite paclitaxel and spirulina nanoparticle were better than paclitaxel and warrant promote preclinical research as a promising agent in the chemotherapy for gastric malignancy, as demonstrated over, the development of the growth xenografts in mice was postponed after the application of paclitaxel.

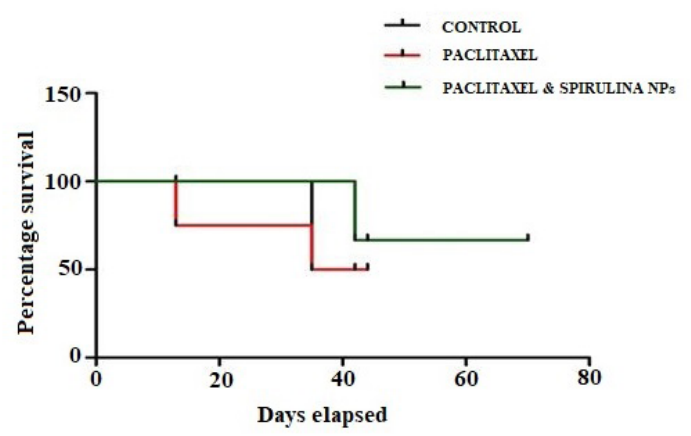

FIGURE 4 - Kaplan-Meier survival curves of gastric cancer xenograft bearing mice. The day when tumor tissues were inoculated into the mice is defined as day 0 . The statistical significance of differences in the survival curves between the groups were evaluated using the log-rank test.
In principle the free paclitaxel, the quicker leeway of the free paclitaxel from the body is decreased the measure of the medication entering in the tumor cells, while for nanoscale paclitaxel and spirulina nanoparticles, in which the improved antitumour viability was appeared, which might be caused by the protecting impact, of the PEG crown, Combining with upgraded endocytosis productivity. It ought to be noticed that the paclitaxel details utilized in the investigation indicated diverse requests of the antitumor action in vitro and in vivo.

The slices for hematoxylin and eosin staining were obtained along the margin of the excised tumor xenografts where the blood supply was theoretically better than that within the tumor. As shown in figure 5, the areas of tissue necrosis (indicated by straight arrows) significantly increased in the paclitaxel \& spirulina nanoparticle group compared with the others, which further demonstrated the enhanced antitumor efficacy of pure paclitaxel. In addition, a few neutrophils, lymphocytes, and macrophages were found around or within the necrotic area of the tumor tissue sections, which is evidence of phagocytosis and a clearance reaction after cell apoptosis (Jersmann et al., 2003, Ikeda et al., 2005).

TABLE II - Median and Mean survival time

\begin{tabular}{lccccc}
\hline Group & $\mathbf{N}$ & Volume $\left(\mathbf{m m}^{2}\right)$ & TIR (\%) & Net weight (g) & TIR (\%) \\
\hline Control & 14 & $562 \pm 264^{\mathbf{\Delta}}$ & & $0.52 \pm 0.18^{\mathbf{\Lambda}}$ & \\
Paclitaxel & 14 & $321 \pm 175^{* \mathbf{\Delta}}$ & 39.8 & $0.41 \pm 0.21^{* \mathbf{\Lambda}}$ & 22.2 \\
$\begin{array}{l}\text { Paclitaxel \& spirulina } \\
\text { nanoparticles }\end{array}$ & 15 & $154 \pm 132^{*}$ & 73.2 & $0.24 \pm 0.12^{*}$ & 62.8 \\
\hline
\end{tabular}

Volume data: ${ }^{*} p<0.01$ versus control; $\boldsymbol{\Delta}_{p}<0.01$ versus paclitaxel \& spirulina nanoparticles; $p=0.812$ paclitaxel Versus paclitaxel $\&$ spirulina nanoparticles; Weight data: ${ }^{*} p<0.01$ versus control; ${ }^{\mathbf{\Delta}} p<0.01$ versus paclitaxel \& spirulina nanoparticles; $p=0.017$, control versus paclitaxel; $p=0.097$. paclitaxel versus paclitaxel $\&$ spirulina nanoparticles. 

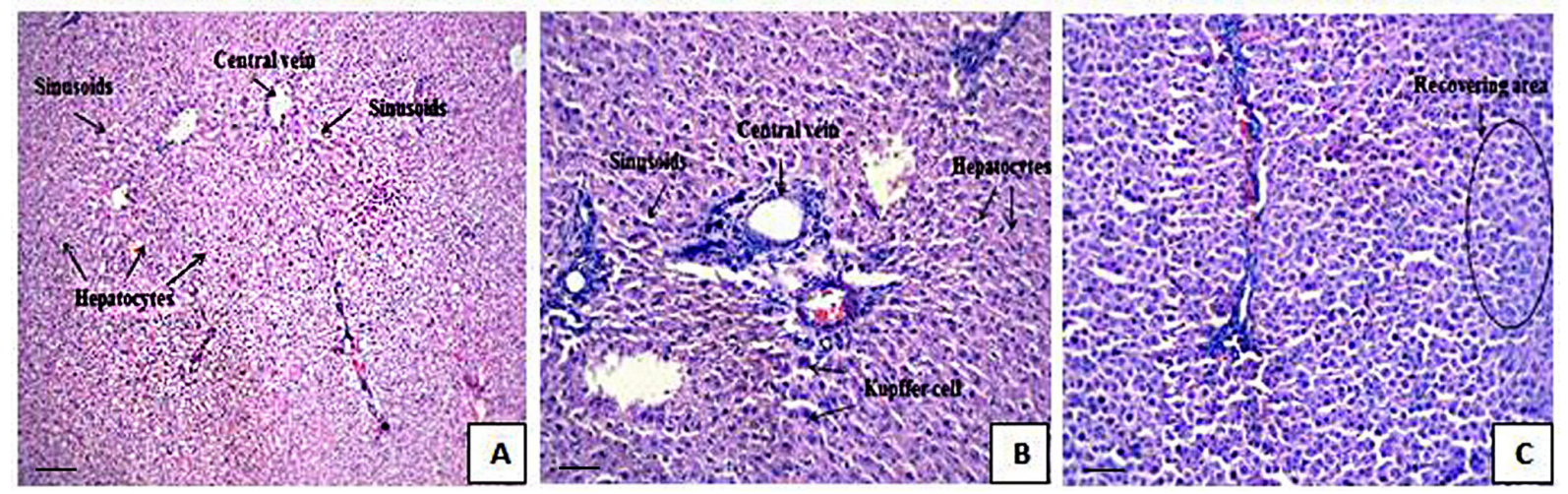

FIGURE 5 - Histopathological Examination. Tumor slices stained with hematoxylin and eosin. The slices were prepared from the margin of xenografts, and the necrotic area in the paclitaxel \& spirulina nanoparticle group is significantly increased compared with that of the other groups. (A. Normal control) sheets of prominent hepatocytes, sinusoids \& central vein; (B. Tumor control)hepatic congestion at sinusoids and the portal vessel, pericentre globular micro-steatosis, Kupffer cell proliferation and hepatocyte diffuse necrosis; (C. paclitaxel \& spirulina nano particle treated) No hepatic congestion at sinusoids and the portal vessel, pericentre globular micro-steatosis, No Kupffer cell proliferation and no hepatocyte diffuse necrosis. (Scale bar $50 \mu \mathrm{m}$ ).

\section{DISCUSSION}

When paclitaxel reduces the toxic side effects against normal tissues it could be said as successful chemotherapeutic agent, and it should avoid the intolerable solvents. To increase the localization of the drug towards the tumor and minimizing the systemic toxicity different methods were deisgned and expected that the spirulina could be stabilizing effectively the nanoparticles which are loaded with the paclitaxel and also when co encapsulated with the paclitaxel. (Harada et al., 2009, Rogosnitzky, Danks, 2011).

The anticancer activity of the paclitaxel towards the gastric cancer cells could be enhanced by spirulina. Hence the co encapsulation of the paclitaxel and spirulina into the Nanoparticles will be showing better stability than paclitaxel loaded nanoparticles, and retainity of the anticancer efficiency of paclitaxel of paclitaxel and spirulina. When the cytotoxicity studies have done the invitro cytotoxicity test indicating that nearly nontoxic dose of spirulina is significantly increasing the cytotoxicity of the paclitaxel. In the current and previous studies the results like the paclitaxel and spirulina nanoparticles having the potential anticancer efficiency in gastrointestinal cancer.

Here in the trademark pharmacodynamics and pharmacokinetics of intra tumoral conveyance decides the restorative adequacy of the medication paclitaxel and spirulina nanoparticles. The In vivo assessment in the mice demonstrates when conveyed in the Intra tumoral infusion it demonstrated the antitumor potential of paclitaxel and spirulina nanoparticles. As it is said to be the site particular conveyance to the tumor knob, the co-drug stacked nanoparticles will be in like manner inciting the higher local drug concentration than systemic delivery. Furthermore, subsequently this is exceptionally sensible that the medication which is realeased from the intra tumorally conveyed nanoparticles will stay for quite a while in the interstitial space of the tumor contrasted with the typical tissue and excerts an extended tumor wiping out impact locally. Henceforth the paclitaxel and spirulina by intra tumoral application can't accomplish as high as introductory focus as free medications and the sustained release is equipped for conveying the medication to its antitumor viability continually. With a specific end goal to the further extend the parameters of this current research, further development on the basis of multidrug application by nanoparticles will be completely checked on in human orthotopic xenograft models.

\section{CONCLUSION}

This study concludes that the paclitaxel and spirulina are stable co drug loaded nanoparticles, which are formed by the amphillic methoxy PEG PCL block copolymers. Here providing the invitro studies in which the superior cytotoxicity of paclitaxel and 
spirulina nanoparticles in a Dose-dependent pattern against the MKN45 cells, And whereas the invivo study in an established MKN45 transplanted mice model, the evaluation showed that the paclitaxel and spirulina nanoparticles are deliverd to the intra tumour, and was effectively exhibited the significant improved antitumour efficacy and substantially increased the overall survival.

\section{ACKNOWLEDGEMENT}

The authors express their gratitude to the Department of Pharmacology and Pharmaceutics, Vels Institute of Science Technology and Advanced Studies, Chennai, India.

\section{REFERENCES}

Bajaj G, Yeo Y. Drug delivery systems for intraperitoneal therapy. Pharm Res. 2010;27(5):735-738.

Bissery MC, Nohynek G, Sanderink GJ, Lavelle F. Docetaxel (Taxotere): a review of preclinical and clinical experience. Part I: preclinical experience. Anticancer Drugs. 1995;6(3):339-355.

Diaz JF, Andreu JM. Assembly of purified GDP-tubulin into microtubules induced by taxol and taxotere: reversibility, ligand stoichiometry, and competition. Biochemistry. 1993;32(11):2747-2755.

Dorr RT. Pharmacology and toxicology of Cremophor EL diluent. Ann Pharmacother. 1994;28(Suppl 5):11-14.

Harada K, Ferdous T, Itashiki Y, Takii M, Mano T, Mori Y et al. Effects of cepharanthine alone and in combination with fluoropyrimidine anticancer agent, S-1, on tumor growth of human oral squamous cell carcinoma xenografts in nude mice. Anticancer Res. 2009;29(4):1263-70.

Idani H, Matsuoka J, Yasuda T, Kobayashi K, Tanaka N. Intratumoral injection of doxorubicin (Adriamycin) encapsulated in liposome inhibits tumor growth, prolongs survival time and is not associated with local or systemic side effects. Int $\mathrm{J}$ Cancer. 2000;88(4):645-651.
Ikeda R1, Che XF, Yamaguchi T, Ushiyama M, Zheng CL, Okumura $\mathrm{H}$ et al. Cepharanthine potently enhances the sensitivity of anticancer agents in K562 cells. Cancer Sci. 2005;96(6):372-6.

Isobe Y, Nashimoto A, Akazawa K, Oda I, Hayashi K, Miyashiro I et al. Gastric cancer treatment in Japan: 2008 annual report of the JGCA nationwide registry. Gastric Cancer. 2011;14(4):301-16.

Jemal A, Bray F, Center MM, Ferlay J, Ward E, Forman D. Global cancer statistics. CA Cancer J Clin. 2011;61(2):69-90.

Jersmann HP, Ross KA, Vivers S, Brown SB, Haslett C, Dransfield I. Phagocytosis of apoptotic cells by human macrophages: analysis by multiparameter flow cytometry. Cytometry A. 2003;51(1):7-15.

Li X, Li R, Qian X, Ding Y, Tu Y, Guo R et al. Superior antitumor efficiency of cisplatin-loaded nanoparticles by intratumoral delivery with decreased tumor metabolism rate. Eur J Pharm Biopharm. 2008;70(3):726-734.

Li X1, Lu X, Xu H, Zhu Z, Yin H, Qian X et al. Paclitaxel/ tetrandrine coloaded nanoparticles effectively promote the apoptosis of gastric cancer cells based on oxidation therapy. Mol Pharm. 2012;9(2):222-229.

Maeda H. The enhanced permeability and retention (EPR) effect in tumor vasculature: the key role of tumor-selective macromolecular drug targeting. Adv Enzyme Regul. 2001;41:189-207.

Rogosnitzky M1, Danks R. Therapeutic potential of the biscoclaurine alkaloid, cepharanthine, for a range of clinical conditions. Pharmacol Rep. 2011;63(2):337-47.

Yamada J1, Kitayama J, Tsuno NH, Yamashita H, Miyato H, Soma D et al. Intra-peritoneal administration of paclitaxel with non-animal stabilized hyaluronic acid as a vehicle: a new strategy against peritoneal dissemination of gastric cancer. Cancer Lett. 2008;272(2):307-15.

Received for publication on $06^{\text {th }}$ December 2018 Accepted for publication on $12^{\text {nd }}$ July 2019 